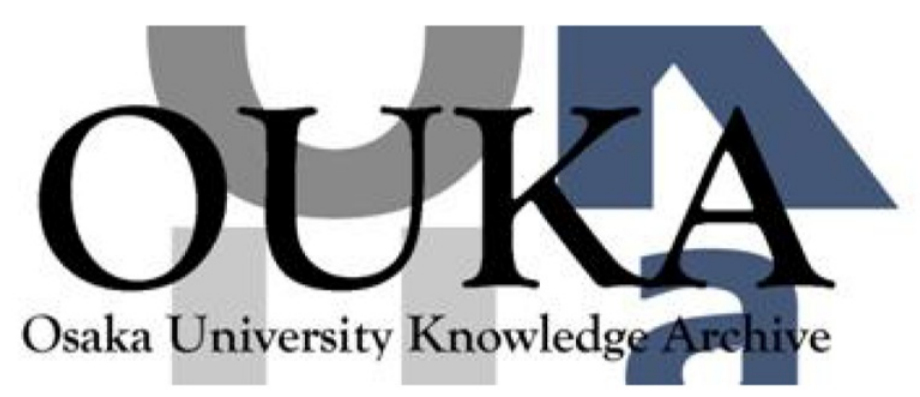

\begin{tabular}{|c|l|}
\hline Title & $\begin{array}{l}\text { Mode-selective resonance ultrasound } \\
\text { spectroscopy of a layered parallelepiped }\end{array}$ \\
\hline Author(s) & $\begin{array}{l}\text { Ogi, Hirotsugu; Heyliger, Paul; Ledbetter, } \\
\text { Hassel et al. }\end{array}$ \\
\hline Citation & $\begin{array}{l}\text { Journal of the Acoustical Society of America. } \\
108(6) \text { p. 2829-p. } 2834\end{array}$ \\
\hline Issue Date & $2000-12$ \\
\hline oaire:version & VoR \\
\hline URL & $\begin{array}{l}\text { https://hdl. handle. net/11094/84146 } \\
\text { Copyright 2000 Acoustical Society of America. } \\
\text { This article may be downloaded for personal use } \\
\text { only. Any other use requires prior permission } \\
\text { of the author and the Acoustical Society of } \\
\text { America. }\end{array}$ \\
\hline rights \\
\hline Note & \begin{tabular}{l} 
\\
\hline
\end{tabular} \\
\hline
\end{tabular}

Osaka University Knowledge Archive : OUKA

https://ir. Library. osaka-u. ac. jp/

Osaka University 


\title{
Mode-selective resonance ultrasound spectroscopy of a layered parallelepiped
}

\author{
Hirotsugu Ogi \\ Graduate School of Engineering Science, Osaka University, Machikaneyama 1-3, Toyonaka, \\ Osaka 560-8531, Japan \\ Paul Heyliger \\ Department of Civil Engineering, Colorado State University, Fort Collins, Colorado 80523 \\ Hassel Ledbetter and Sudook Kim \\ Materials Science and Engineering Laboratory, National Institute of Standards and Technology, \\ Boulder, Colorado 80303
}

(Received 1 March 2000; revised 11 September 2000; accepted 15 September 2000)

\begin{abstract}
The resonance frequencies of mechanical free vibration of a three-layer material calculated by a discrete-layer model and measured by acoustic-resonance methods were compared. The material was composed of an aluminum parallelepiped sandwiched by two stainless-steel parallelepipeds. The discrete-layer model developed here used linear Lagrange basis functions through the layered dimension and continuous global power-series basis functions in the plane perpendicular to the layer thickness. Using such a basis function for the layer-thickness direction allows discontinuity in the elastic properties across the interface between dissimilar layers. The resonance frequencies were measured using two methods: mode-selective electromagnetic acoustic resonance (EMAR) and resonance ultrasound spectroscopy (RUS). The measurements agreed with the calculations typically within $1 \%$. The EMAR method allows the selective detection of vibrational modes possessing particular displacement patterns. This selectivity was supported by the model calculation. Thus, using the EMAR method makes mode identification clear and this is essential for developing and improving the model calculation of such a complicated structure. Internal friction was also measured by the two acoustic methods, which reveals the mechanical-contact effect on the internal-friction measurement in the RUS method. (C) 2000 Acoustical Society of America.
\end{abstract}

[S0001-4966(00)04812-8]

PACS numbers: 43.35.Cg, 43.35.Zc [SGK]

\section{INTRODUCTION}

Layered systems are prominent in modern engineering components and applications. A layered geometry can be formed as part of shape or stiffness control, to adjust material properties for different applications, or to provide different properties in particular directions. In all cases, the characteristics of both local and global component response become significantly more complicated than for a single homogeneous layer. This is especially so in the calculation of laminate-material elastic properties. In this study, we present comparisons between a laminate-continuum model that takes into account the distinct material properties of each individual layer and experimental observations for the problem of unrestrained free mechanical vibration. Such a comparison is first made here, and it is critical to establish the model calculation to represent layered systems and to eventually adopt such a model to compute the properties for a material of unknown constitution, including a thin film on a substrate.

Concerning measurement of the resonance frequency of free vibration, we principally use electromagnetic acoustic resonance (EMAR) ${ }^{1-4}$ which is an acoustically contactless method and can select particular vibrational modes of interest by controlling the deformation symmetry of vibration using the Lorentz-force mechanism. This mode-selective prin- ciple provides a significant advantage for making exact correspondence between the measured and calculated resonance frequencies. Along with the resonance frequency, we measure internal friction by the free-decay method of EMAR. Also, we use the usual resonance ultrasound spectroscopy (RUS) $)^{5,6}$ for the resonance frequencies and internal friction, and discuss the effect of contacting transduction in the RUS method by comparing with the contactless EMAR results.

For the calculation of resonance frequencies, we adopt the discrete-layer model developed by Heyliger. ${ }^{7}$ The calculated resonance frequencies essentially showed good agreement with the measured frequencies, but agreed less well for higher modes.

\section{MATERIAL}

We used a diffusion-bonded three-layer parallelepiped, consisting of stainless steel (SS304), aluminum (A13004), and stainless-steel (SS304) polycrystals. (Two stainless-steel parallelepipeds sandwiched the aluminum parallelepiped.) The dimensions of each layer are given in Table I. We measured elastic constants using the Pulse-echo method and density using Archimedes method before bonding. They are given in Table II. 
TABLE I. Dimensions of the layered specimen in $\mathrm{mm}$

\begin{tabular}{ccccc}
\hline \hline$x$ & $y$ & $z_{1}(\mathrm{SS} 304)$ & $z_{2}(\mathrm{Al3004})$ & $z_{3}$ (SS304) \\
\hline 2.982 & 3.988 & 0.394 & 1.477 & 0.465 \\
\hline \hline
\end{tabular}

\section{DISCRETE-LAYER MODEL}

The resonance frequencies of natural vibration of solids are obtainable by solving the equations of motion with boundary conditions at free surfaces. However, since the direct solutions are unobtainable for rectangular parallelepipeds, we seek approximate solutions by solving the weak form of the equations of motion. Assuming periodic particle motion and using Cartesian coordinates, where $u, v$, and $w$ denote displacements along $x, y$, and $z$ axes, respectively, the weak form of the equations of motion for a linear solid is reduced as ${ }^{7,8}$

$$
\begin{gathered}
\int_{V}\left\{\rho \omega^{2}(u \delta u+v \delta v+w \delta w)-\left[C_{11} \frac{\partial u}{\partial x} \frac{\partial \delta u}{\partial x}+C_{12} \frac{\partial u}{\partial x} \frac{\partial \delta v}{\partial y}\right.\right. \\
+C_{13} \frac{\partial u}{\partial x} \frac{\partial \delta w}{\partial z}+C_{12} \frac{\partial v}{\partial y} \frac{\partial \delta u}{\partial x}+C_{22} \frac{\partial v}{\partial y} \frac{\partial \delta v}{\partial y} \\
+C_{23} \frac{\partial v}{\partial y} \frac{\partial \delta w}{\partial z}+C_{13} \frac{\partial w}{\partial z} \frac{\partial \delta u}{\partial x}+C_{23} \frac{\partial w}{\partial z} \frac{\partial \delta v}{\partial y} \\
+C_{33} \frac{\partial w}{\partial z} \frac{\partial \delta w}{\partial z}+C_{44}\left(\frac{\partial v}{\partial z}+\frac{\partial w}{\partial y}\right)\left(\frac{\partial \delta v}{\partial z}+\frac{\partial \delta w}{\partial y}\right) \\
+C_{55}\left(\frac{\partial u}{\partial z}+\frac{\partial w}{\partial x}\right)\left(\frac{\partial \delta u}{\partial z}+\frac{\partial \delta w}{\partial x}\right)+C_{66}\left(\frac{\partial u}{\partial y}+\frac{\partial v}{\partial x}\right) \\
\left.\left.\quad \times\left(\frac{\partial \delta u}{\partial y}+\frac{\partial \delta v}{\partial x}\right)\right]\right\} d V+\int_{S} \bar{T}_{k} \delta u_{k} d S=0 .
\end{gathered}
$$

Hence, $\delta$ denotes the variational operator, $C_{i j}$ the elastic stiffnesses, $\omega$ the periodic angular frequency of vibration, and $\bar{T}_{k}$ the components of the specified surface tractions. $V$ and $S$ denote the volume and surface occupied by and bounding the solid, respectively.

Our model for computing estimates of the natural frequencies of vibration follows, with minor variation, the approach of Heyliger. ${ }^{7}$ The nature of the approximations used to solve Eq. (1) for layered parallelepipeds must take into account the behavior of the elastic stress and strain fields through the interfaces as it undergoes deformation during resonance. Specifically, traction continuity across a dissimilar material interface that is perpendicular to the $z$ axis requires that the stress components $\sigma_{z z}, \sigma_{x z}, \sigma_{y z}$ must all be continuous across the interface. Also, displacements $u, v$, and $w$ must be continuous. Therefore, the fact that there is a

TABLE II. Elastic constants and mass density of the individual material measured before bonding.

\begin{tabular}{lccc}
\hline \hline & $\mathrm{C}_{11}(\mathrm{GPa})$ & $\mathrm{C}_{44}(\mathrm{GPa})$ & $\rho\left(\mathrm{kg} / \mathrm{m}^{3}\right)$ \\
\hline Al3004 & 110.7 & 26.16 & 2699 \\
$\mathrm{SS} 304$ & 261.4 & 77.40 & 7489 \\
\hline \hline
\end{tabular}

discontinuity in the shear moduli $\mu$ implies that the shear strain components are discontinuous. For example, we consider the strain component $\gamma_{x z}$ defined as

$$
\gamma_{x z}=\frac{\partial u}{\partial z}+\frac{\partial w}{\partial x}
$$

It is clear that if this function is discontinuous at the interface, then the displacement component $u$ must have a jump in slope with respect to $z$ as one travels through the thickness of the specimen. Hence, conventional Legendre polynomials or power series, which have been used with outstanding results in many past studies ${ }^{9,10}$ to compute the resonance frequency of free vibration, are unacceptable candidates for vibration studies of layered media.

In the discrete-layer approximation, the dependence of the displacements on the $z$ coordinate is separated from the functions in $x$ and $y$, and it is allowed to have a break in slope with respect to the layer (or $z$ ) coordinate. This allows for global functions in $x$ and $y$ that are continuous and have continuous derivatives in the $x-y$ plane, but still maintains the necessary physical features in $z$ required by the mismatch in material properties. Hence, approximations for the displacement $u$ can be expressed as ${ }^{11}$

$$
\begin{aligned}
u(x, y, z, t) & =\sum_{j=1}^{n} U_{j}(x, y, t) \bar{\Psi}_{j}^{u}(z) \\
& =\sum_{i=1}^{m} \sum_{j=1}^{n} U_{j i}(t) \Psi_{i}^{u}(x, y) \bar{\Psi}_{j}^{u}(z) .
\end{aligned}
$$

As seen in Ref. 11, approximations for $v$ and $w$ can take similar forms. $n$ denotes the number of layers and $m$ the number of in-plane basis functions $\Psi_{i}(x, y)$. The approximations for each of the three field quantities are constructed in such a way as to separate the dependence in the plane from that in the direction perpendicular to the interface. In the thickness direction, we used one-dimensional Lagrangian interpolation polynomials for $\bar{\Psi}_{j}(z)$. For the in-plane approximations, we used power series $x^{p} y^{q}(p, q=0,1,2, \ldots)$. For a parallelepiped with $n$ layers, $(n-1)$ is the number of subdivisions through the parallelepiped thickness, and $U_{j i}$, for example, denotes the coefficient of $u$ at height $j$ corresponding to the $i$ th in-plane approximation function. ${ }^{11}$

Substituting these approximations into Eq. (1), collecting the coefficients of the variations of the displacements, and placing the results in matrix form yields the result

$$
\omega^{2}[\mathbf{M}]\{\xi\}=[\mathbf{K}]\{\xi\} .
$$

Here, $[\mathbf{M}]$ denotes the mass matrix, associated with the kinetic energy of the system, and $[\mathbf{K}]$ denotes the stiffness matrix, associated with the potential energy of the system. $\{\xi\}$ denotes the vector of constants that multiplies the displacement functions. Hence, the final problem results in the form of an eigenvalue problem.

In general, it is possible to split the groupings of the approximation functions to coincide with specific modal displacement patterns to reduce the size of the computational problem that must be solved. This behavior has been previously discussed by Ohno ${ }^{9}$ and Visscher et al. ${ }^{12}$ One of the 
TABLE III. Group structure for layered specimen.

\begin{tabular}{|c|c|c|c|}
\hline \multirow[b]{2}{*}{ Group } & \multicolumn{3}{|c|}{ Displacement } \\
\hline & & $x$ & $\mathrm{y}$ \\
\hline \multirow[t]{3}{*}{1} & $u$ & $E$ & E \\
\hline & $v$ & $O$ & $\mathrm{O}$ \\
\hline & $w$ & $O$ & $\mathrm{E}$ \\
\hline \multirow[t]{3}{*}{2} & $u$ & $E$ & $\mathrm{O}$ \\
\hline & $v$ & $O$ & $\mathrm{E}$ \\
\hline & $w$ & $O$ & $\mathrm{O}$ \\
\hline \multirow[t]{3}{*}{3} & $u$ & $O$ & $\mathrm{O}$ \\
\hline & $v$ & $E$ & $\mathrm{E}$ \\
\hline & $w$ & $E$ & $\mathrm{O}$ \\
\hline \multirow[t]{3}{*}{4} & $u$ & $O$ & $\mathrm{E}$ \\
\hline & $v$ & $E$ & $\mathrm{O}$ \\
\hline & $w$ & $E$ & E \\
\hline
\end{tabular}

disadvantages of our discrete-layer model is that we lose some of the splitting properties in the $z$ direction when we use the discontinuous Lagrangian shape functions. However, it is still possible to make this split for the functions in the $x-y$ plane. Instead of the eight groupings allowed for homogeneous orthotropic or isotropic materials, ${ }^{9}$ four groups are allowable. ${ }^{7}$ We give the displacement patterns corresponding to the four vibration groups in Table III. For example, group 1 includes vibration modes whose displacement component $u$ is an even function about $x$ and $y$. Hence, if power series are being used, the functions used would appear as $1, x^{2}, y^{2}$, $x^{2} y^{2}, x^{4}$, etc. As our freedom in choosing the odd or even character of our functions in $z$ has vanished when we select piecewise linear functions, no grouping is allowed in $z$. However, and more important, we may thus model the break in displacement gradient. Our model can also be directly applied to specimens with no symmetry about the $x-y$ plane. Concerning the material used in the present study, we can discuss the $z$-direction symmetry because of nearly symmetrical structure in the layered thickness.

In practical terms for our calculations, we used a total of 15 layers to model the 3 primary material layers. In plane, we use terms up to and including 8th order for each of the 3 displacement components. This leads to a total number of unknowns equal to $(15+1)(8)(8)(3)=3072$ in Eq. (4).

Splitting the functions as we do allows for solving 4 problems of size 768 , a much more appealing proposition.

\section{ACOUSTIC-RESONANCE MEASUREMENTS}

Resonance ultrasound spectroscopy, or RUS, is widely adopted to study elastic properties of solids. ${ }^{5,6}$ The specimen can be a sphere, cylinder, rectangular parallelepiped, or other regular shape, which is put between two ultrasonic transducers; one transducer generates a sinusoidal continuous-wave oscillation and the other detects the amplitude of mechanical vibrations at the contact point. By measuring the amplitude as a function of the driving frequency, we obtain a spectrum consisting of many resonance peaks, which are input to an inverse calculation to find the complete set of elastic con-

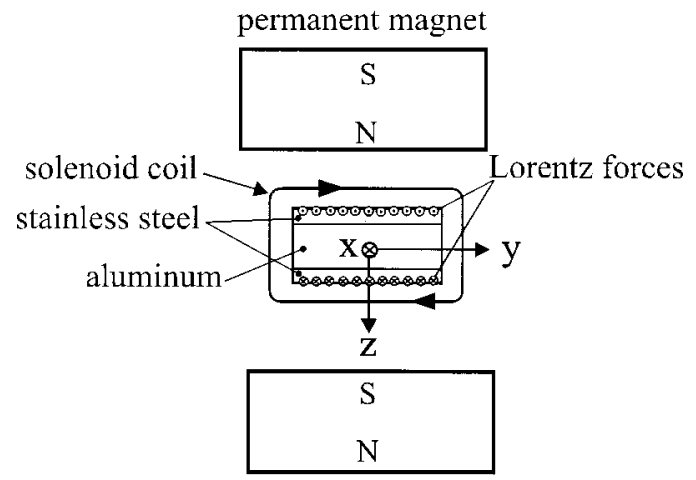

FIG. 1. EMAR-measurement setup for group 1.

stants of the material. The RUS method can determine all independent elastic constants with a single frequency scan for a single small specimen.

For a rectangular parallelepiped of orthorhombic or higher elastic symmetry, we can divide free mechanical vibrations into eight groups depending on the deformation symmetry. ${ }^{9}$ The computation of the resonance frequencies is based on such groupings. Therefore, successful use of the RUS method requires exact correspondence between the measured and calculated resonance frequencies, otherwise the resultant elastic properties have no physical significance. In the usual RUS method, however, such mode identification is not straightforward because all of the eight vibration
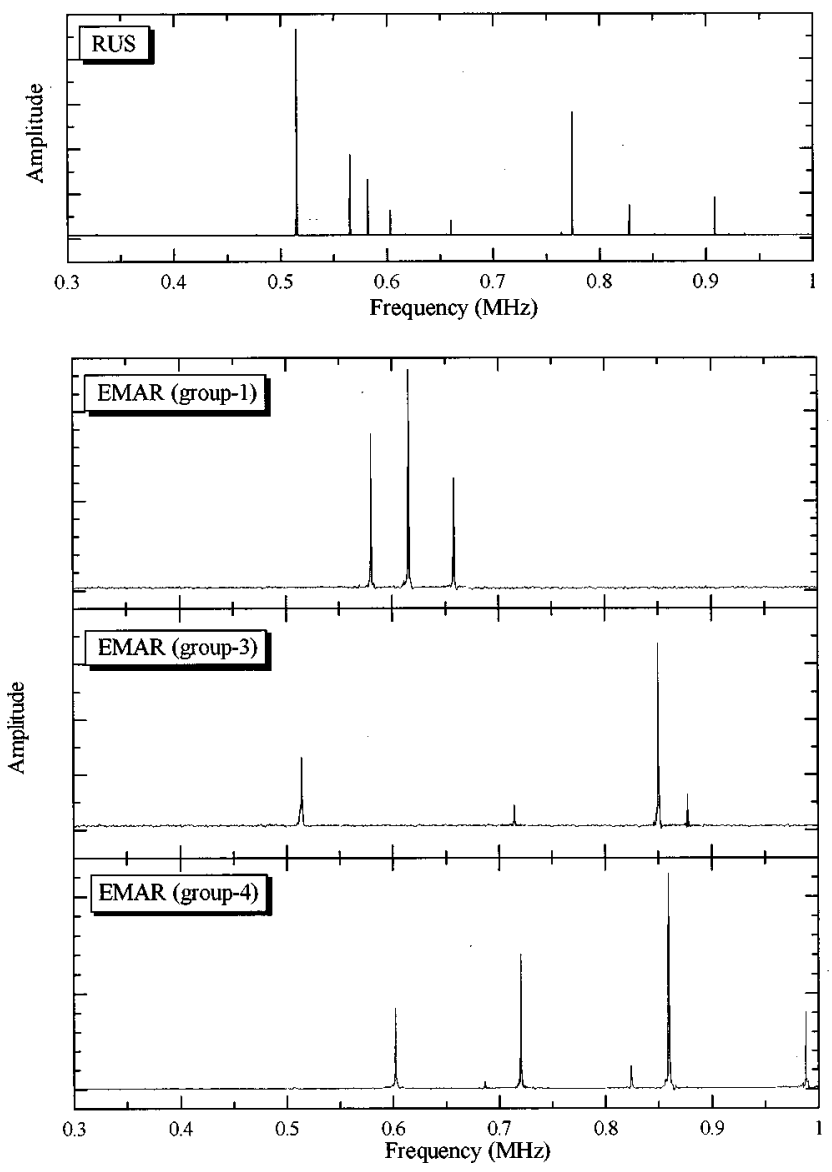

FIG. 2. RUS resonance spectrum and EMAR resonance spectra measured by the three different measurement setups. 
TABLE IV. Calculated resonance frequencies $f_{\text {calc }}$ by the discrete-layer model; measured resonance frequencies by the EMAR $\left(f_{\mathrm{EMAR}}\right)$ and RUS $\left(f_{\mathrm{RUS}}\right)$ methods; and internal friction measured by the EMAR $\left(Q^{-1}\right.$ EMAR $)$ and RUS $\left(Q_{\text {RUS }}^{-1}\right)$ methods.

\begin{tabular}{|c|c|c|c|c|c|c|c|c|}
\hline \multirow[b]{2}{*}{ Group } & \multirow{2}{*}{$\begin{array}{c}f_{\text {calc }} \\
(\mathrm{MHz})\end{array}$} & \multirow{2}{*}{$\begin{array}{c}f_{\mathrm{RUS}} \\
(\mathrm{MHz})\end{array}$} & \multirow{2}{*}{$\begin{array}{l}f_{\text {EMAR }} \\
(\mathrm{MHz})\end{array}$} & \multicolumn{3}{|c|}{ Diff } & \multirow{2}{*}{$\begin{array}{l}Q^{-1}{ }_{\mathrm{RUS}} \\
\left(10^{-4}\right)\end{array}$} & \multirow{2}{*}{$\begin{array}{c}\left.Q^{-1}{ }_{\text {EMAR }}^{-4}\right) \\
\left(10^{-4}\right.\end{array}$} \\
\hline & & & & $f_{\text {calc }}-f_{\text {RUS }}$ & $f_{\text {calc }}-f_{\text {EMAR }}$ & $f_{\text {RUS }}-f_{\text {EMAR }}$ & & \\
\hline 2 & 0.330618 & 0.327540 & $\cdots$ & 0.93 & $\cdots$ & $\cdots$ & 6.41 & $\cdots$ \\
\hline 4 & 0.444477 & $\cdots$ & $\cdots$ & $\cdots$ & $\ldots$ & $\ldots$ & $\ldots$ & $\ldots$ \\
\hline 2 & 0.475870 & 0.477209 & $\cdots$ & -0.28 & $\cdots$ & $\cdots$ & 1.49 & $\cdots$ \\
\hline 1 & 0.483995 & 0.480354 & $\cdots$ & 0.75 & $\cdots$ & $\cdots$ & 5.4 & $\cdots$ \\
\hline $3^{b}$ & 0.511266 & 0.515126 & 0.513816 & -0.75 & -0.50 & 0.25 & 2.11 & 0.83 \\
\hline $1^{\mathrm{a}}$ & 0.577386 & 0.565280 & 0.580562 & 2.10 & -0.55 & -2.63 & 2.91 & 0.86 \\
\hline 2 & 0.578794 & 0.582057 & $\ldots$ & -0.56 & $\ldots$ & $\ldots$ & 1.74 & $\ldots$ \\
\hline $4^{c}$ & 0.603155 & 0.603177 & 0.602211 & 0.00 & 0.16 & 0.16 & 1.53 & 1.98 \\
\hline $1^{\mathrm{a}}$ & 0.619723 & 0.617616 & 0.615400 & 0.34 & 0.70 & 0.36 & 2.77 & 1.46 \\
\hline 4 & 0.655140 & 0.656059 & $\ldots$ & -0.14 & $\ldots$ & $\ldots$ & 1.96 & $\ldots$ \\
\hline $1^{\mathrm{a}}$ & 0.667090 & 0.660323 & 0.658002 & 1.01 & 1.36 & 0.35 & 1.36 & 1.48 \\
\hline 3 & 0.688171 & 0.684499 & $\cdots$ & 0.53 & $\cdots$ & $\cdots$ & 1.16 & $\cdots$ \\
\hline $4^{c}$ & 0.690143 & 0.688024 & 0.687352 & 0.31 & 0.40 & 0.1 & 1.3 & 1.48 \\
\hline $3^{b}$ & 0.716305 & $\cdots$ & 0.714567 & $\cdots$ & 0.24 & $\cdots$ & $\ldots$ & 1.74 \\
\hline $4^{c}$ & 0.721124 & $\cdots$ & 0.720579 & $\cdots$ & 0.08 & $\ldots$ & $\cdots$ & 0.99 \\
\hline 1 & 0.761508 & 0.752794 & $\cdots$ & 1.14 & $\cdots$ & $\cdots$ & 0.8 & $\cdots$ \\
\hline 2 & 0.768587 & 0.764560 & $\cdots$ & 0.52 & $\cdots$ & $\cdots$ & 0.78 & $\cdots$ \\
\hline 2 & 0.781888 & 0.774563 & $\ldots$ & 0.94 & $\cdots$ & $\cdots$ & 1.71 & $\ldots$ \\
\hline 3 & 0.806825 & $\cdots$ & $\cdots$ & $\cdots$ & $\cdots$ & $\cdots$ & $\cdots$ & $\cdots$ \\
\hline 4 & 0.831013 & 0.825310 & $\cdots$ & 0.69 & $\cdots$ & $\cdots$ & 2.21 & $\cdots$ \\
\hline 1 & 0.831996 & 0.828144 & $\ldots$ & 0.46 & $\ldots$ & $\ldots$ & 1.15 & $\ldots$ \\
\hline $4^{c}$ & 0.832514 & 0.839802 & 0.824117 & -0.88 & 1.01 & 1.9 & 1.17 & 0.97 \\
\hline 3 & 0.845328 & $\cdots$ & $\cdots$ & $\cdots$ & $\cdots$ & $\cdots$ & $\cdots$ & $\cdots$ \\
\hline $3^{b}$ & 0.855176 & 0.851729 & 0.849814 & 0.40 & 0.63 & 0.23 & 1.23 & 1.17 \\
\hline $4^{c}$ & 0.866047 & 0.861784 & 0.859193 & 0.49 & 0.79 & 0.3 & 1.71 & 1.66 \\
\hline 4 & 0.869413 & 0.868335 & $\cdots$ & 0.12 & $\cdots$ & $\cdots$ & 2.05 & $\cdots$ \\
\hline 3 & 0.885307 & 0.878115 & $\cdots$ & 0.81 & $\cdots$ & $\cdots$ & 2.04 & $\cdots$ \\
\hline $3^{b}$ & 0.890438 & $\ldots$ & 0.877694 & $\cdots$ & 1.43 & $\ldots$ & $\ldots$ & 1.41 \\
\hline 2 & 0.915726 & 0.908094 & $\ldots$ & 0.83 & $\ldots$ & $\cdots$ & 1.18 & $\ldots$ \\
\hline 1 & 0.918189 & 0.921349 & $\cdots$ & -0.34 & $\cdots$ & $\cdots$ & 1.54 & $\cdots$ \\
\hline 2 & 0.933194 & $\cdots$ & $\cdots$ & $\cdots$ & $\cdots$ & $\cdots$ & $\cdots$ & $\cdots$ \\
\hline 3 & 0.935763 & 0.936009 & $\ldots$ & -0.03 & $\ldots$ & $\ldots$ & 1.69 & $\ldots$ \\
\hline 4 & 0.943766 & $\ldots$ & $\ldots$ & $\cdots$ & $\cdots$ & $\cdots$ & $\ldots$ & $\cdots$ \\
\hline $4^{c}$ & 0.999864 & $\cdots$ & 0.986506 & $\cdots$ & 1.34 & $\cdots$ & $\cdots$ & 1.6 \\
\hline 1 & 1.006263 & $\cdots$ & $\ldots$ & $\cdots$ & $\cdots$ & $\cdots$ & $\cdots$ & $\ldots$ \\
\hline 2 & 1.017014 & $\cdots$ & $\ldots$ & $\ldots$ & $\cdots$ & $\cdots$ & $\cdots$ & $\ldots$ \\
\hline $1^{\mathrm{a}}$ & 1.036230 & $\cdots$ & 1.023532 & $\cdots$ & 1.23 & $\cdots$ & $\cdots$ & 2.5 \\
\hline 4 & 1.037997 & $\cdots$ & $\cdots$ & $\cdots$ & $\cdots$ & $\cdots$ & $\cdots$ & $\cdots$ \\
\hline $4^{\mathrm{c}}$ & 1.056968 & $\cdots$ & 1.040538 & $\cdots$ & 1.55 & $\cdots$ & $\cdots$ & 1.4 \\
\hline 2 & 1.060712 & $\cdots$ & $\ldots$ & $\cdots$ & $\cdots$ & $\cdots$ & $\cdots$ & $\ldots$ \\
\hline $3^{b}$ & 1.072812 & $\cdots$ & 1.067099 & $\cdots$ & 0.53 & $\cdots$ & $\cdots$ & 1.72 \\
\hline 2 & 1.093049 & $\cdots$ & $\cdots$ & $\cdots$ & $\cdots$ & $\cdots$ & $\cdots$ & $\cdots$ \\
\hline 1 & 1.122839 & $\ldots$ & $\ldots$ & $\ldots$ & $\ldots$ & $\ldots$ & $\ldots$ & $\ldots$ \\
\hline $4^{c}$ & 1.129148 & $\ldots$ & 1.112440 & $\ldots$ & 1.48 & $\cdots$ & $\ldots$ & 1.28 \\
\hline $1^{\mathrm{a}}$ & 1.132007 & $\ldots$ & 1.115818 & $\ldots$ & 1.43 & $\ldots$ & $\ldots$ & 0.49 \\
\hline $3^{b}$ & 1.154532 & $\cdots$ & 1.111587 & $\cdots$ & 3.72 & $\ldots$ & $\ldots$ & 1.84 \\
\hline
\end{tabular}

a $u$-odd-in- $z$ modes.

${ }^{\mathrm{b}} v$-odd-in- $z$ modes.

${ }^{c} w$-odd-in- $z$ modes.

groups are simultaneously excited and many resonance peaks overlap, which causes inability to track overtones. The best way for mode identification would be to independently produce only one group of vibrations, filtering out the others. This is possible with EMAR, as described below.

In the typical EMAR-measurement setup, the specimen is inserted in a solenoid coil located between two permanentmagnet blocks (see Fig. 1). We drive the solenoid coil with high-power rf bursts to induce eddy currents near the surface region of the specimen and then the Lorentz forces through interaction between eddy currents and the static magnetic field from the permanent magnets. The Lorentz forces oscillate with the same frequency as the driving bursts, causing the mechanical vibration. After the excitation, the same coil detects the vibrations through the reversed-Lorentz-force mechanism, the received signals are fed to the superheterodyne phase-sensitive detectors, and the amplitude spectrum at the operating frequency is determined. A single frequency 
scan provides many resonance peaks (but fewer than in the RUS case), and the resonance frequencies are determined by Lorentzian-function fitting.

Such an EMAR configuration enables us to easily change the Lorentz-force direction and then select particular vibration modes. For example, in Fig. 1, the magnetic field is applied along the $z$ axis and the Lorentz forces occur on the $x-y$ faces along the $x$ axis. The Lorentz forces produce the $x$ component of displacement $u$. The displacement is most effectively detected by the same coil through the reversedLorentz-force mechanism when $u$ is an even function about $x$ and $y$, and an odd function about $z$. Among the vibration modes classified into four groups in Table III, only the vibration modes having an odd deformation pattern of $u$ along the $z$ axis in group 1 satisfy this condition. Thus, only those vibration modes should be generated and detected with this configuration. Similarly, we can independently generate and detect the $v$-odd-in- $z$ modes in group 3 and $w$-odd-in- $z$ modes in group 4, with different EMAR configurations (see Ref. 3).

For internal friction, we measured the amplitude decay after EMAR excitation with a tone burst. The amplitude freedecay curve provides the internal friction at the resonance frequency. ${ }^{13}$

We also made the usual RUS measurement using two pinducers sandwiching the specimen. In this case, we determined internal friction from the resonance-peak width. The typical measurement reproducibility of EMAR and RUS was $10^{-6}$ to $10^{-5}$ for resonance frequencies and $10^{-5}$ to $10^{-4}$ for internal friction.

\section{RESULTS AND DISCUSSION}

We show the resonance spectra measured by the RUS and EMAR methods in Fig. 2. The different EMAR measurement configurations produced different spectra, as expected. Table IV gives the measured and calculated resonance frequencies. In the model calculation, we investigated the eigenvector to predict the deformation pattern for each mode and found the particular vibration modes that $u$ is odd about $z$ in group 1, $v$ is odd about $z$ in group 3, and $w$ is odd about $z$ in group 4 . Those modes were faultlessly detected by the three different EMAR configurations described above, indicating that the mode-selective principle of EMAR worked well. The differences between calculated frequencies and frequencies measured with EMAR were less than $1 \%$ for lower modes, but larger for higher modes. What is the most important observation here is that the closest pair between the measurement and calculation did not always provide exact mode correspondence. For example, the seventh mode of group 4 detected by EMAR occurred at $0.824117 \mathrm{MHz}$ and the closest frequency predicted from the model was the sixth mode of group 4. However, this is an undetectable mode with EMAR because it fails to satisfy the condition of detection of EMAR. If we had no information about the symmetry of the excited modes, we may have incorrectly identified this mode. The same thing could occur more easily for higher modes because of larger differences between the calculations and measurements. Thus, the mode-selective or modeidentified method is very significant and essential in
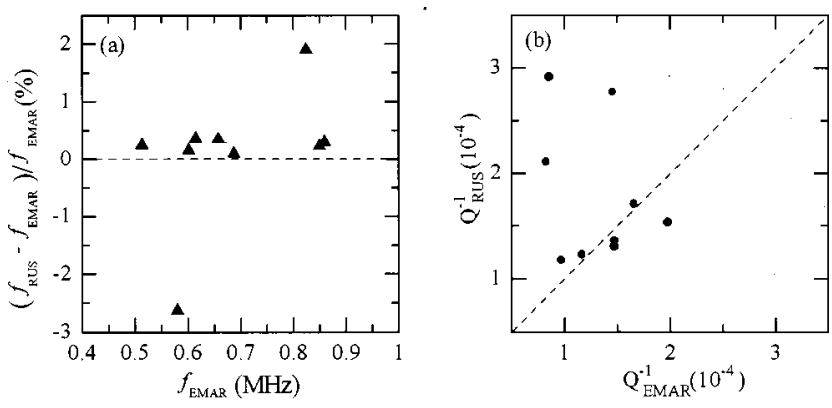

FIG. 3. Comparisons of RUS and EMAR measurements for (a) resonance frequencies and (b) internal friction of corresponding modes.

resonance-ultrasound-spectroscopy study. The fact that there were larger differences between calculated and measured frequencies of higher modes suggests that we need to use a larger number of discrete layers. Also, the discrete-layer model is based on the Ritz method, which typically gives less accurate frequencies as the mode number increases.

The RUS spectrum showed a better signal-to-noise ratio than the EMAR spectra. The RUS measurements agreed with calculations typically with $1 \%$ difference. But, we failed to identify the higher-frequency modes because of small amplitude and peak overlapping. An important observation is that the RUS method gave higher resonance frequencies than those of the EMAR method for almost all modes, as seen in Table IV and Fig. 3(a). We attribute this to the applied force of sandwiching transducers which is needed for mechanical coupling in the RUS method. Such an applied force constrains the specimen and prevents it from ideal free vibration, leading to upward shifts in resonance frequencies. ${ }^{14}$

Comparisons of internal friction measured by EMAR and RUS are shown in Table IV. Figure 3(b) also demonstrates the coupling effect. For most matching modes, RUS internal friction was noticeably larger than EMAR internal friction. This indicates the energy loss into the contacting transducers in the RUS method, which was pointed out in a previous study. ${ }^{3}$

\section{CONCLUSIONS}

(1) We have presented the first comparisons between the measurement and calculation of a free-vibration problem of a layered-parallelepiped material. The agreements between measured and calculated resonance frequencies were typically within $1 \%$. This difference is expected to decrease by increasing the number of the discrete layers in the calculation.

(2) The discrete-layer model explained the nature of vibration well. The deformation pattern of a vibration estimated by the model calculation was supported by the modeselective EMAR measurements. Such an exact mode identification is absolutely necessary for developing and improving the model.

(3) The comparisons between the RUS and EMAR results show the effect of contact coupling, even for the relatively weak point contacting of RUS made in this study. 


\section{ACKNOWLEDGMENTS}

The contribution of the second author was completed during an Alexander von Humboldt Fellowship at the Staatliche Materialprüfungsanstalt (MPA) at the University of Stuttgart. This support is gratefully acknowledged. Professor V. Kinra (Texas A \& M) contributed the studied material.

${ }^{1}$ M. Hirao and H. Ogi, "Electromagnetic Acoustic Resonance and Materials Characterization," Ultrasonics 35, 413-421 (1997).

${ }^{2}$ W. Johnson, "Ultrasonic Damping in Pure Aluminum at Elevated Temperatures,"’ J. Appl. Phys. 83, 2462-2468 (1998).

${ }^{3}$ H. Ogi, H. Ledbetter, S. Kim, and M. Hirao, "Contactless Mode-Selective Resonance Ultrasound Spectroscopy: Electromagnetic Acoustic Resonance," J. Acoust. Soc. Am. 106, 660-665 (1999).

${ }^{4}$ H. Ogi, K. Takashima, H. Ledbetter, M. Dunn, G. Shimoike, M. Hirao, and P. Bowen, "Elastic Constants and Internal Friction of an SiC-FiberReinforced Ti-Alloy-Matrix Crossply Composite: Measurement and Theory," Acta Mater. 47, 2787-2796 (1999).

${ }^{5}$ J. Maynard, "Resonant Ultrasound Spectroscopy," Phys. Today 49, 26-31 (1996).

${ }^{6}$ A. Migliori and J. L. Sarrao, Resonant Ultrasound Spectroscopy (Wiley, New York, 1997).
${ }^{7}$ P. R. Heyliger, "Traction-Free Vibration of Layered Elastic and Piezoelectric Rectangular Parallelepipeds,' J. Acoust. Soc. Am. 107, 12351245 (2000)

${ }^{8}$ H. F. Tiersten, Linear Piezoelectric Plate Vibrations (Plenum, New York, 1969).

${ }^{9}$ I. Ohno, "Free Vibration of A Rectangular Parallelepiped Crystal and Its Application to Determination of Elastic Constants of Orthorhombic Crystals,"' J. Phys. Earth 24, 355-379 (1976).

${ }^{10}$ A. Migliori, J. L. Sarrao, W. M. Visscher, T. M. Bell, M. Lei, Z. Fisk, and R. G. Leisure, "Resonant Ultrasound Spectroscopy technique for measurement of the elastic moduli of solids," Physica B 183, 1-24 (1993).

${ }^{11}$ J. N. Reddy, "A Generalization of Two-Dimensional Theories of Laminated Composite Plates," Commun. Appl. Numer. Methods 3, 173-180 (1987)

${ }^{12}$ W. M. Visscher, A. Migliori, T. M. Bell, and R. A. Reinert, "On the Normal Modes of Free Vibration of Inhomogeneous and Anisotropic Elastic Objects,", J. Acoust. Soc. Am. 90, 2154-2162 (1991).

${ }^{13}$ H. Ogi, M. Hirao, and T. Honda, "Ultrasonic Attenuation and Grain Size Evaluation Using Electromagnetic Acoustic Resonance," J. Acoust. Soc. Am. 98, 458-464 (1995).

${ }^{14}$ Y. Sumino, I. Ohno, T. Goto, and M. Kumazawa, "Measurement of Elastic Constants and Internal Friction on Single-Crystal MgO by Rectangular Parallelepiped Resonance,'” J. Phys. Earth 24, 263-273 (1976). 